Notes \& Tips

\title{
Escherichia coli bactofection using Lipofectamine
}

\author{
Kumaran Narayanan ${ }^{\mathrm{a}, \mathrm{b}, *}$, Choon Weng Lee ${ }^{\mathrm{c}}$, Aurelian Radu ${ }^{\mathrm{d}}$, Edmund Ui Hang Sim ${ }^{\mathrm{e}}$ \\ ${ }^{a}$ School of Science, Monash University, 46150 Selangor Darul Ehsan, Malaysia \\ ${ }^{\mathrm{b}}$ Department of Genetics and Genomic Sciences, Icahn School of Medicine at Mount Sinai, New York, NY 10029, USA \\ ${ }^{\mathrm{c}}$ Institute of Biological Sciences, University of Malaya, 50603 Kuala Lumpur, Malaysia \\ ${ }^{\mathrm{d}}$ Department of Developmental and Regenerative Biology, Icahn School of Medicine at Mount Sinai, New York, NY 10029, USA \\ e Department of Molecular Biology, Faculty of Resource Science and Technology, Universiti Malaysia Sarawak, 94300 Kota Samarahan Sarawak, Malaysia
}

\section{A R T I C L E I N F O}

\section{Article history:}

Received 24 March 2013

Received in revised form 6 April 2013

Accepted 8 April 2013

Available online 19 April 2013

\section{Keywords:}

Bactofection

E. coli

Invasin

DNA delivery

Transfection reagent

Lipofectamine

\begin{abstract}
A B S T R A C T
Successful gene delivery into mammalian cells using bactofection requires entry of the bacterial vector via cell surface integrin receptors followed by release of plasmid DNA into the cellular environment. We show, for the first time, that addition of the DNA transfection reagent Lipofectamine improves entry of invasive Escherichia coli into HeLa cells and enhances up to 2.8-fold green fluorescent protein (GFP) expression from a reporter plasmid. The addition of Lipofectamine may be applicable to other bacterial vectors to increase their DNA delivery efficiency into mammalian cells.
\end{abstract}

(c) 2013 Elsevier Inc. All rights reserved.
Successful gene expression using bactofection requires entry of the bacterial vector via integrin receptors followed by release of plasmid DNA into the cellular environment. Integrins are transmembrane surface receptors found on the surface of most mammalian cells $[1,2]$. They are involved in cell-cell adhesion and enable interaction of mammalian cells and components of the extracellular matrix [1,2].

Integrin receptors have been exploited previously for targeting plasmid DNA into cells as part of nonviral gene transfer strategies. Remarkably, integrin-targeting efficiency of the DNA was improved by the simple addition of DNA transfection reagents, resulting in considerably higher gene expression. In one example, $\beta$-galactosidase reporter expression from integrin-targeted peptide/DNA complexes was enhanced from approximately $10 \%$ to more than $50 \%$ in melanoma cells (A375 M) and monkey kidney epithelial cells (COS-7) when Lipofectin was added [3]. In another work, the addition of the cationic liposome DOTAP or the polymer polyethylenimine to plasmid DNA attached to a histone H1derived DNA binding domain that targets the integrin receptor increased luciferase expression from the plasmid by 10 - to 20 -fold in HeLa cells compared with plasmid combined with the transfection reagents alone [4]. Although the actual mechanism is still

\footnotetext{
* Corresponding author at: School of Science, Monash University, 46150 Selangor Darul Ehsan, Malaysia. Fax: +6 0355146364.

E-mail address: kumaran.narayanan@monash.edu (K. Narayanan).
}

unknown, these studies demonstrated convincingly that addition of DNA transfection reagents could enhance gene delivery via integrin receptors.

Based on these observations, we hypothesized that DNA transfection reagents could increase the gene delivery efficiency of a bactofection vector that uses the integrin receptor. To test this hypothesis, we investigated the effect of the widely used, commercially available Lipofectamine reagent on the invasive asd $^{-}$Escherichia coli DH10B vector that we developed previously [5]. This vector is a diaminopimelic acid-requiring auxotroph derived from the commercially available DH10B strain that is adapted to invade and deliver genes into mammalian cells when the Yersinia pseudotuberculosis invasin gene is coexpressed [5]. To investigate the effect of Lipofectamine on bactofection, we tested the ability of the vector to (i) internalize into cells and (ii) express the green fluorescent protein (GFP) ${ }^{1}$ reporter gene from a plasmid.

In the internalization experiment, increasing concentrations of Lipofectamine were combined with a constant number of either invasive or noninvasive bacteria and presented to cells. The invasive E. coli vector contained the $Y$. pseudotuberculosis invasin gene, facilitating its entry using integrin receptors [5,6]. The noninvasive E. coli, isogenic to the invasive strain but without the invasin gene, was used to determine background internalization [5].

\footnotetext{
${ }^{1}$ Abbreviations used: GFP, green fluorescent protein; MOI, multiplicity of infection.
} 